\title{
THE 1989 OUTBURST OF V404 CYGNI: A VERY UNUSUAL X-RAY NOVA
}

\author{
R. M. Wagner \\ Department of Astronomy, The Ohio State University \\ S. Starrfield \\ IGPP and Theoretical Division, Los Alamos National Laboratory \\ and Department of Physics and Astronomy, Arizona State University \\ A. Cassatella and R. Gonzalez-Riestra \\ ESA IUE Observatory, VILSPA \\ T. J. Kreidl \\ Lowell Observatory \\ S. B. Howell \\ Planetary Science Institute \\ R. M. Hjellming and X.-H. Han \\ National Radio Astronomy Observatory \\ C. Shrader \\ IUE Observatory, Goddard Space Flight Center \\ G. Sonneborn \\ Laboratory for Astronomy and Solar Physics, Goddard Space Flight Center \\ G. W. Collins and R. Bertram \\ Department of Astronomy, The Ohio State University \\ M. W. Buie and H. E. Bond \\ Space Telescope Science Institute \\ J. Johnson, T. Harrison, and R. D. Gehrz \\ School of Physics and Astronomy, University of Minnesota \\ F. A. Córdova \\ Earth and Space Sciences Division, Los Alamos National Laboratory \\ P. Szkody and B. Margon \\ Department of Astronomy, University of Washington \\ J. MacDonald \\ Department of Physics and Astronomy, University of Delaware \\ R. Fried \\ Braeside Observatory
}

\begin{abstract}
On May 22, 1989 the Japanese Ginga Team discovered a new X-ray source that was cataloged as GS $2023+338$. This source was subsequently identified as coincident in position with a previously known nova cataloged as V404 Cygni. Its last recorded outburst was in 1938 when it rose to about 12 th mag. Spectroscopic data were obtained and confirmed the nature of the outburst. Additional ground based data were obtained by us at CTIO and the VLA. The X-ray behavior of this object has been reported to be very unusual and it reached a peak of about 17 crab about one week after discovery. Since then it has varied widely in magnitude at all wavelengths at which it has been studied. We present a brief summary of the observations that have been obtained up to the time of the meeting and shortly thereafter.
\end{abstract}



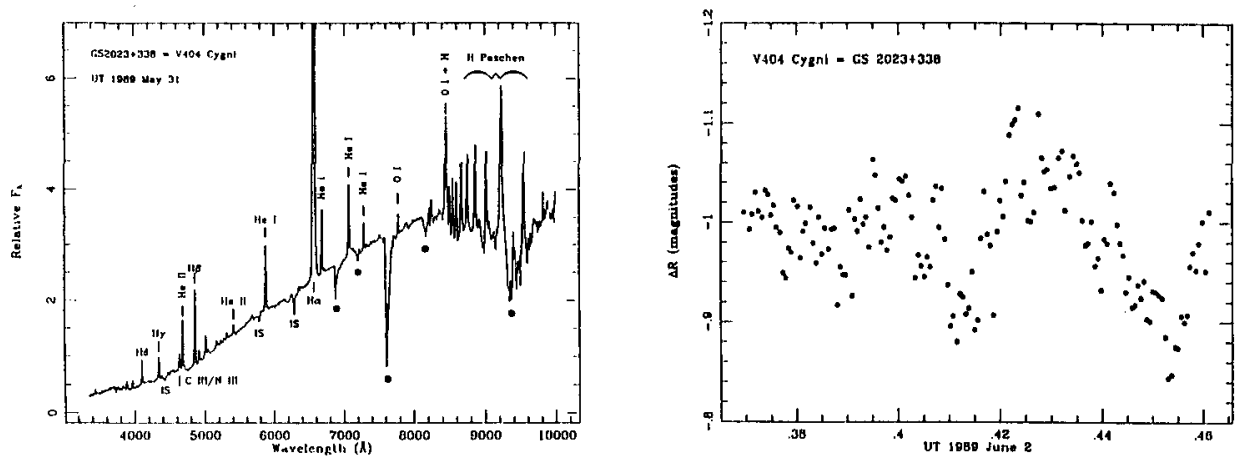

Figure 1 - Optical spectrum of V404 Cyg obtained by R.M. Wagner on UT 1989 May 31 at the Perkins 1.8-m Telescope using Ohio State University CCD Spectrograph. The spectrum is dominated by strong hydrogen and helium emission lines superposed on a reddened continuum.

Figure 2 - Light curve of V404 Cyg obtained by T.J. Kreidl and S.B. Howell on UT 1989 June 2 at the Perkins Telescope using the Lowell Observatory direct imaging RCA CCD camera. Note the strong flickering on time scales of minutes or shorter.
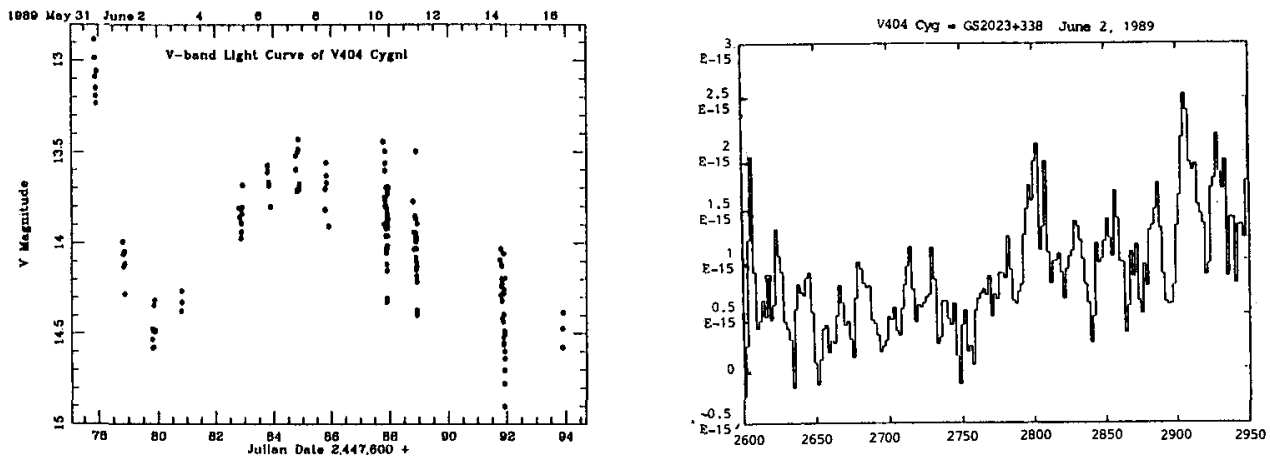

Figure 3 - V-band light curve of V404 Cyg from late May through mid-June recorded by R. Fried using a computer controlled $0.4-\mathrm{m}$ telescope and photoelectric photometry system.

Figure 4 -Optimally extracted IUE spectrum obtained by Angelo Cassatella and Chris Shrader on UT June 2, 1989. It is the result of a combined US1+VILSPA 792 minute exposure. It shows a weak continuum and $\mathrm{Mg}$ II in emission. This is the first UV spectrum of an X-ray nova in outburst. 